\title{
Ulcerações Labiais Associadas a Comportamento Obsessivo-Compulsivo: Relato de Caso
}

\author{
Letícia Logullo7, Helio G Rocha Neto², Karla Calaca Kabbach Prigenzi ${ }^{3}$, Sandra Lopes Mattos e Dinato ${ }^{4}$ \\ 'Especializanda em Dermatologia do Centro Universitário Lusíada - UNILUS - Hospital Guilherme Álvaro, São Paulo, Brasil \\ ${ }^{2}$ Mestre em Psiquiatria pela Universidade Federal do Rio de Janeiro. Professor do Centro Universitário Lusíada - UNILUS, São Paulo, \\ Brasil \\ ${ }^{3}$ Especialista em Anatomia Patológica e Citopatologia pela Sociedade Brasileira de Patologia e Citopatologia. Doutoranda em \\ Patologia pela Universidade Federal de São Paulo- UNIFESP. Professora de Patologia do Centro Universitário Lusíada - UNILUS, São \\ Paulo, Brasil \\ ${ }^{4}$ Doutora em Dermatologia pela Faculdade de Medicina da Universidade de São Paulo (FMUSP). Professora do Centro Universitário \\ Lusíada - UNILUS, São Paulo, Brasil
}

RESUMO - As lesões auto-infligidas compreendem modificações cutâneas induzidas por um desvio do comportamento e demandam o envolvimento de diferentes profissionais da saúde. São mais comuns entre as mulheres de meia idade. Relata-se o caso clínico de um homem de 42 anos com diagnóstico de lesão ulcerada nos lábios superior e inferior, secundária a comportamento obsessivo-compulsivo. Os achados histopatológicos foram inespecíficos e os resultados de exames laboratoriais realizados não apresentaram alterações. Optou-se pelo acompanhamento conjunto entre a Psiquiatria e a Dermatologia, com seguimento de sessões de psicoterapia, mas o paciente acabou por abandonar o tratamento. Ressalta-se a importância do conhecimento das psicodermatoses pelo dermatologista, visando o diagnóstico e a terapêutica precoces, o que permitirá a prevenção de lesões destrutivas e mutiladoras.

PALAVRAS-CHAVE - Comportamento Autodestrutivo; Lábio; Transtorno Obsessivo-Compulsivo/complicações; Úlcera Cutânea/etiologia.

\section{Lip Ulcerations Associated with Obsessive- Compulsive Behavior: Case Report}

ABSTRACT - Self-inflicted lesions include skin alterations caused by an abnormal behavior. They demand interdisciplinary involvement of health professionals. They are most common among middle-aged women. We report the case of a 42-year-old man with a diagnosis of an ulcerated lesion on the upper and lower lips, secondary to obsessive-compulsive behavior. Histopathological findings were non-specific and the results of laboratory tests performed showed no alterations. Joint evaluation by Psychiatry and Dermatology was initiated, with follow-up sessions of psychotherapy. Patient chose to abandon treatment. The importance of recognizing psychodermatoses for the dermatologist is emphasized, aiming at the early diagnosis and therapeutics, which will allow the prevention of destructive and mutilating lesions.

KEYWORDS - Lip; Obsessive-Compulsive Disorder/complications; Self-Injurious Behavior; Skin Ulcer/etiology.

\section{INTRODUÇÃO}

As lesões auto-infligidas compreendem modificações cutâneas induzidas por um desvio do comportamento e demandam o envolvimento de diferentes profissionais da saúde. A incidência relatada é de 0,03\% a 9,4\% em pacientes dermatológicos, com predominância no sexo feminino.' Relata-se o caso de lesão destrutiva dos lábios superior e inferior em paciente com sintomas obsessivo-compulsivos.
Correspondência: Letícia Logullo Dermatologia do Centro Universitário Lusíada UNILUS - Hospital Guilherme Álvaro

Rua Padre João Manuel, 620 apto 81 Cerqueira César

São Paulo, Brasil 01411-000

DOI: https://dx.doi.org/10.29021/spdv.77.1.1018
Recebido/Received 23 Setembro/September 2018 Aceite/Accepted

06 Janeiro/January 2019 


\section{Caso Clínico}

\section{CASO CLÍNICO}

Identificação: Paciente do sexo masculino, caucasiano, 42 anos, solteiro, católico.

História Clínica: Há 6 meses refere o aparecimento de vesículas agrupadas (em "cacho de uvas"), assintomáticas, em lábios superior e inferior, próximo à comissura labial esquerda, com duração de 15 dias. As lesões evoluíram com formação de úlceras na mesma região, com retração importante do lábio superior, permitindo a exposição de alguns dentes da arcada superior à esquerda, mesmo após oclusão dos lábios. Interrogado quanto à manipulação da lesão, referiu fazer limpeza e friç̧ão da região com gaze embebida em soro fisiológico, três vezes ao dia, com finalidade de melhorar o prurido. Após avaliação dermatológi$\mathrm{ca}$, o paciente foi encaminhado para avaliação neurológica e psiquiátrica.

Avaliação dermatológica: Observou-se úlcera de base infiltrada, com bordas esbranquiçadas bem delimitadas e fundo raso, limpo, não secretante, atingindo parte dos lábios superior e inferior e comissura labial esquerda (Fig.s 1 e 2). Foi realizada biópsia dos lábios superior e inferior, cujo exame histopatológico (Fig.s 3 e 4) revelou epiderme com hiperplasia pseudoepiteliomatosa, área de ulceração recoberta por crosta fibrinoleucocitária, exuberante tecido de granulação, fibrose subjacente de padrão cicatricial e

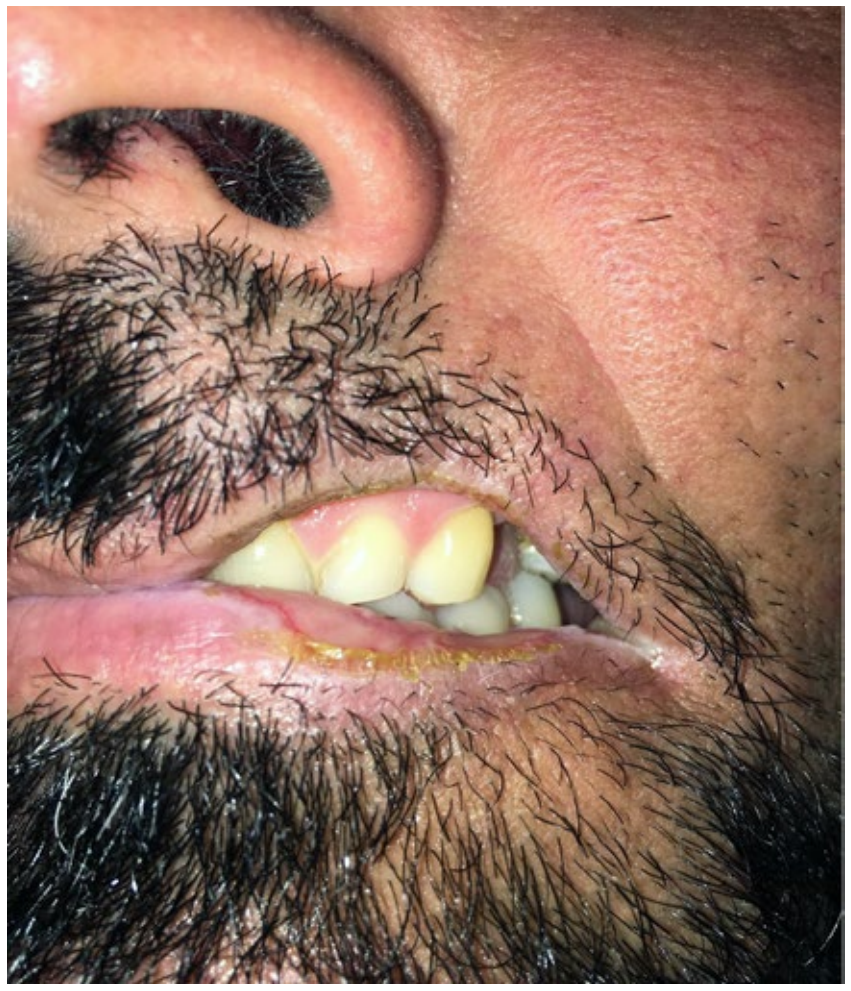

Figura 1 - Lesão de base infiltrada e bordas esbranquiçadas, bem delimitadas, com fundo raso, limpo, não secretante, na metade esquerda dos lábios superior e inferior, com perda de tecido que impede a oclusão completa dos lábios. ausência de sinais de malignidade. Colorações especiais de PAS, Grocott e Ziehl-Neelsen não evidenciaram a presença de fungos ou bacilos.

Avaliação neurológica: Atualmente, sem alterações ao exame neurológico.

Avaliação psiquiátrica: Desde a adolescência relata padrão persistente de preocupação com higiene, limpeza e organização, sem prejuízos funcionais, porém com hábito de vários banhos por dia (de 3 a 5), manutenção do quarto e ambiente de trabalho com alto padrão de limpeza e organização, eventualmente com atraso a compromissos. Apresenta disfemia, devido à presença da extensa lesão labial, que prejudica a fonação. Notado movimento repetitivo de lamber o lábio inferior lesionado, sem outras alterações evidentes. Diagnóstico: Transtorno obsessivo-compulsivo (TOC), subtipo predominantemente compulsivo, com pouco impacto no funcionamento do indivíduo.

Antecedentes Pessoais: Hipertensão arterial (losartan $50 \mathrm{mg} / \mathrm{dia}$ e atenolol $50 \mathrm{mg} / \mathrm{dia}$, indapamida $1,5 \mathrm{mg} / \mathrm{dia}$, nifedipina $2 \mathrm{mg} / \mathrm{dia}$ ), dislipidemia (sinvastatina $40 \mathrm{mg} /$ dia), diabetes mellitus (glicazida $30 \mathrm{mg} /$ dia, metformina $500 \mathrm{mg} / \mathrm{dia}$ ), fator $\mathrm{V}$ de Leiden positivo (varfarina conforme RNI). Acidente vascular cerebral em 2011, observando-se na tomografia computadorizada lesão isquémica cerebelar provavelmente relacionada à síndrome de Wallemberg

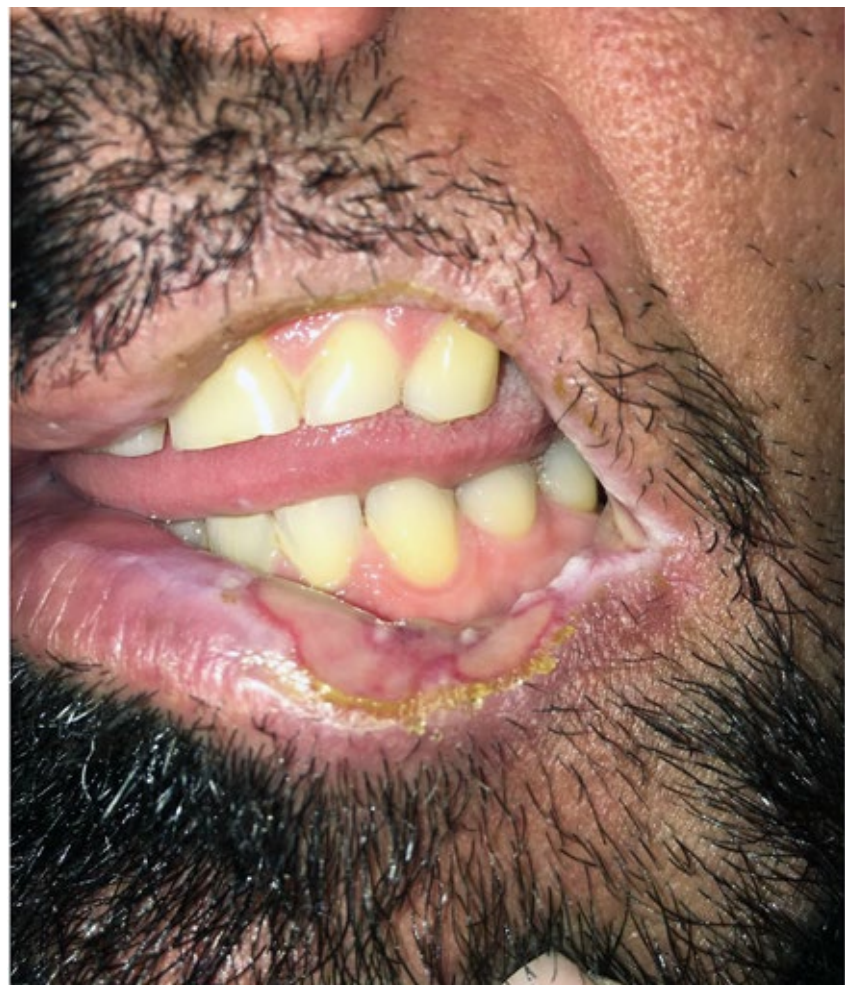

Figura 2 - Detalhe da ulceração do lábio inferior, resultante de movimento repetitivo de lamber e morder o lábio. 


\section{Caso Clínico}

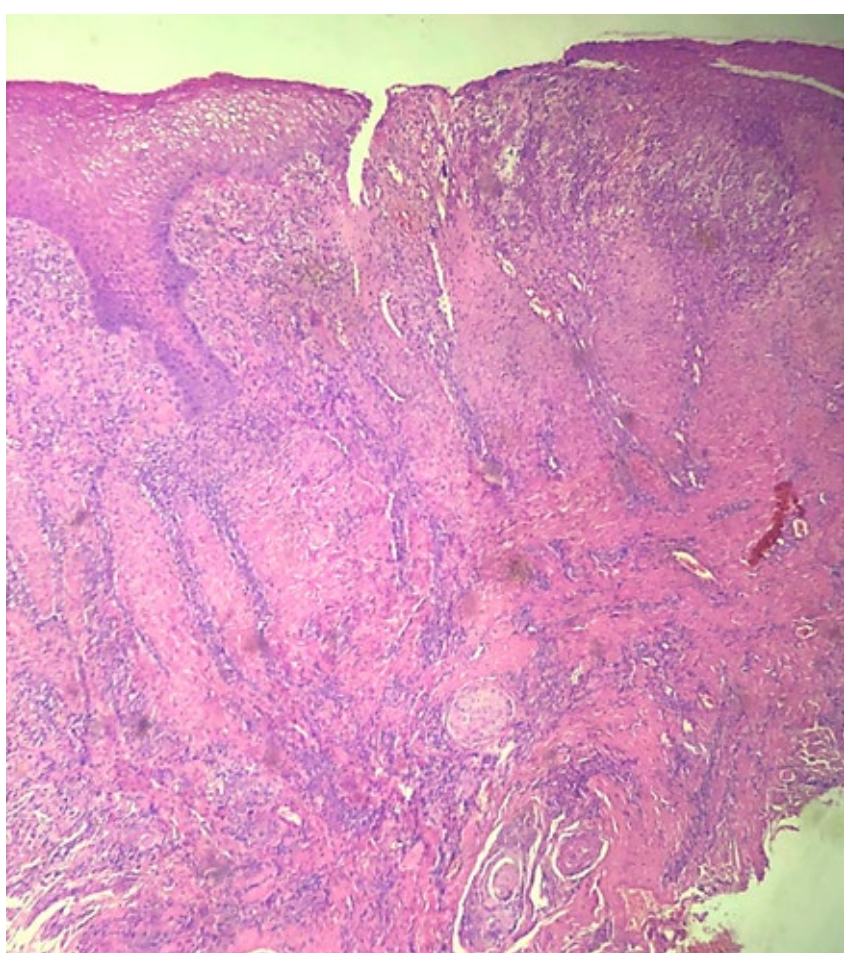

Figura 3 - Imagem histopatológica do lábio evidenciando epiderme com hiperplasia pseudoepiteliomatosa e área de ulceração recoberta por crosta fibrinoleucocitária (HE- 40X).

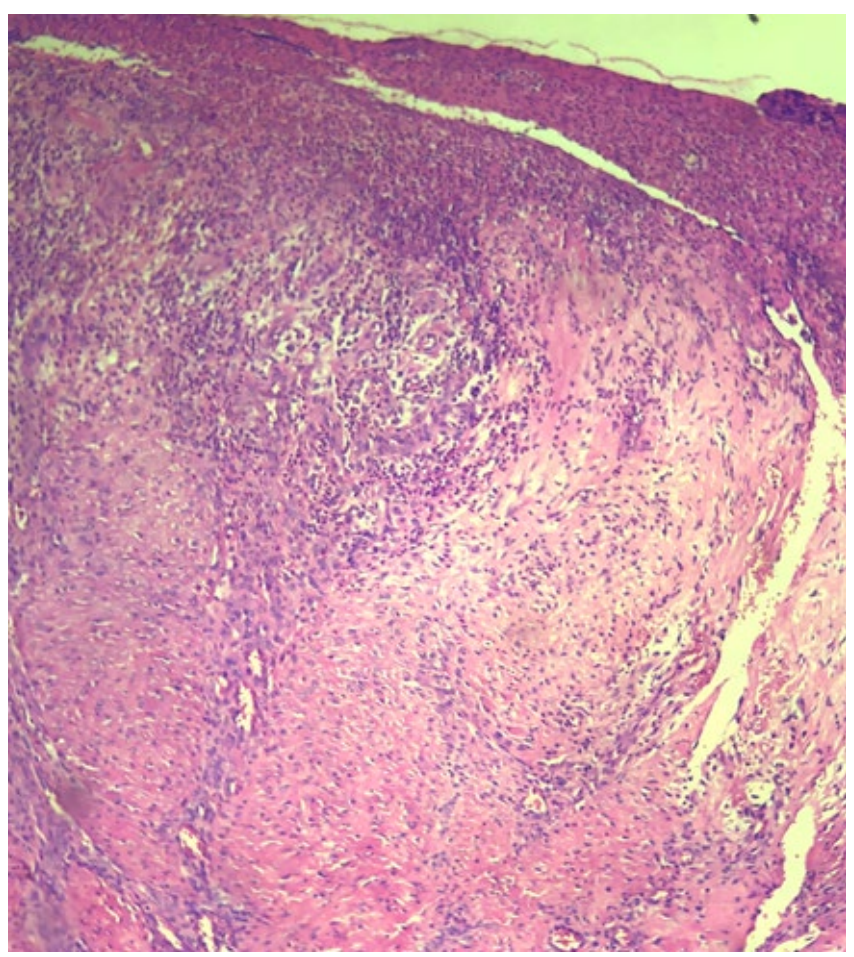

Figura 4 - Detalhe da lesão ulcerada recoberta por crosta fibrinoleucocitária com exuberante tecido de granulação e fibrose subjacente de padrão cicatricial (HE- 40X). (afasia, redução de força em dimídio esquerdo e instabilidade postural) observado na data do acidente vascular cerebral, mas das quais recuperou completamente.

Antecedentes Familiares: Pai falecido aos 47 anos por acidente vascular cerebral hemorrágico. Mãe de 65 anos é hipertensa. Três irmãos hígidos.

Evolução: Optou-se por seguimento ambulatorial com a Psiquiatria, Dermatologia e Endocrinologia. Não foi introduzida medicação psicotrópica no momento, sendo - paciente acompanhado em sessão de psicoterapia de orientação Psicodinâmica Breve semanal. Porém, após 4 semanas abandonou tratamento, ainda sem benefício significativo das lesões cutâneas.

\section{DISCUSSÃO}

As lesões auto-infligidas com comportamento não negado ou oculto subdividem-se em aspectos compulsivos (acne escoriada, tricotilomania e onicofagia) e impulsivos (cortar-se, queimar-se). No espectro compulsivo, os pacientes apresentam o desejo de agir e sentem alívio na atividade. Alguns informam prurido nas lesões, o que dificulta o dignóstico. Embora os pacientes não neguem a indução das lesões, muitos não reconhecem abertamente o problema, como o paciente aqui reportado., ${ }^{1,2}$ Apenas $20 \%$ dos pacientes estão em tratamento e provavelmente o diagnóstico foi tardio. ${ }^{3,4} \mathrm{O}$ tratamento inclui terapia comportamental cognitiva e ou psicoterapia, sozinhas ou combinadas com drogas psicotrópicas, preferencialmente os antidepressivos serotoninérgicos, reservados para casos mais difíceis. ${ }^{1-8} \mathrm{Em}$ muitos casos, o diagnóstico é difícil sem a colaboração de uma equipe multidisciplinar, incluindo Psiquiatra ou Psicodermatologista, além de psicólogos. Todavia, é de grande importância o conhecimento do dermatologista em Psicodermatologia, pois de início muitos pacientes recusam a ida ao Psiquiatra. Os serviços de psicodermatologia são escassos, e a demanda é alta. . $^{6,7}$ Assim, é necessário observação detalhada das lesões elementares, realização de anamnese minuciosa e eventuais exames laboratoriais para segurança no diagnóstico, que é de exclusão.

Conflitos de interesse: Os autores declaram não possuir conflitos de interesse.

Suporte financeiro: $O$ presente trabalho não foi suportado por nenhum subsídio ou bolsa.

Confidencialidade dos dados: Os autores declaram ter seguido os protocolos do seu centro de trabalho acerca da publicação dos dados de doentes.

Direito a privacidade e consentimento escrito: Os autores declaram que pediram consentimento ao representante legal para usar as imagens no artigo.

Consentimento do Doente: Obtido.

Conflicts of interest: The authors have no conflicts of interest to declare.

Financing Support: This work has not received any contribution, grant or scholarship. 


\section{Caso Clínico}

Confidentiality of data: The authors declare that they have followed the protocols of their work center on the publication of data from patients.

Privacy policy and informed consent: The authors declare the patient gave written informed consent for the use of patient's photos in this article.

Patient consent: Obtained.

Proveniência e revisão por pares: Não comissionado; revisão externa por pares

Provenance and peer review: Not commissioned; externally peer reviewed

\section{REFERÊNCIAS}

1. Gieler U, Consoli SG, Tomás-Aragones L, Linder DM, Jemec GB, Poot $F$, et al. Self- inflicted lesions in dermatology: terminology and classification- a posicion paper from the European Society for Dermatology and Psychiatry (ESDaP). Acta Derm Venereol. 2013; 93:4-12. doi: 10.2340/00015555-1506.

2. Roche L, Switzer V, Ramsay B. A retrospective case series of referrals to our psychodermatology clinic 2009-2016. J Eur Acad Dermatol Venereol. 2018; 32e278-9. doi: 10.1111/jdv. 14816.

3. Kestenbaum T. Obsessive-compulsive disorder in dermatology. Semin Cutan Med Surg. 2013; 32:83-7.

4. Zaidens SH. Self- inflicted dermatoses and their psychodynamics. J Nerv Ment Dis. 1951; 113:395-404.

5. Harth W, Mayer K, Linse R. The borderline syndrome in psychosomatic dermatology. Overview and case report. J Eur Acad Dermatol Venereol 2004; 18:503-7. doi: 10.1111/i.1468-3083.2004.00965.x.

6. Azambuja RD. The need of dermatologists, psychiatrists and psychologists joint care in psychodermatology. An Bras Dermatol. 2017; 92:63-71. doi: 10.1590/ abd1806-4841.20175493.

7. Aguilar-Duran S, Ahmed A, Taylor R, Bewley A. How to set up a psychodermatology clinic Clin Exp Dermatol. 2014 ; 39:577-82. doi: 10.1111/ced.12360.

8. Nico MM, Nagano CP, Fernandes JD, Lourenço SV. A destructive ulcer on the lower lip. Clin Exp Dermatol. 2010; 35:e180-1. doi: 10.1111/j.1365-2230.2009.03619.x. 